\title{
Parámetros genéticos para caracteres asociados a la curva de crecimiento de bovinos Tropicarne
}

\section{Genetic parameters for characters associated with the growth curve of Tropicarne cattle}

\author{
Joel Domínguez-Viveros ${ }^{1 *}$, Felipe Alonso Rodríguez-Almeida ${ }^{1}$, Rafael Núñez-Domínguez ${ }^{2}$, Rodolfo \\ Ramírez-Valverde ${ }^{2}$, Juan Ángel Ortega-Gutiérrez ${ }^{1}$ \\ ${ }^{1}$ Facultad de Zootecnia y Ecología; Universidad Autónoma de Chihuahua. Periférico Francisco R. Almada Km 1, CP. 31453, \\ Chihuahua, Chihuahua. \\ ${ }^{2}$ Departamento de Zootecnia; Universidad Autónoma Chapingo. Carretera México - Texcoco km 38.5, CP. 56230, Chapingo, \\ Estado de México. \\ ${ }^{*}$ Autor de correspondencia: joeldguezviveros@yahoo.com.mx, jodominguez@uach.mx
}

Artículo científico recibido: 25 de noviembre de 2015, aceptado: 29 de febrero de 2016

RESUMEN. El objetivo del presente estudio fue estimar parámetros genéticos para el peso adulto (PEA) y tasa de crecimiento (TAC) de bovinos Tropicarne, derivados del modelo no lineal de Brody; así como analizar las posibles relaciones genéticas de estas variables con el peso al destete (PD). Se analizaron 994 datos de PEA (kg) y de TAC $\left(\mathrm{kg}^{-1} \mathrm{mes}^{-1}\right)$ y 1506 datos de PD ajustado a $240 \mathrm{~d}$. Los valores promedio y la desviación estándar $( \pm)$ para PEA, TAC y PD $(\mathrm{kg})$ fueron de $419.1 \pm 94.4,0.0081 \pm 0.0039$ y $206.8 \pm 34.1$, respectivamente. El pedigrí se integró por 2504 individuos. Se realizó un análisis multivariado, a partir de un modelo mixto que consideró como efectos fijos los grupos contemporáneos y la covariable edad de la vaca al parto (lineal y cuadrático); y como aleatorios los efectos genéticos directos y maternos, maternos de ambiente permanente y residuales. Se estimó la heredabilidad directa $\left(h_{a}^{2}\right)$ y materna $\left(h_{m}^{2}\right)$, las correlaciones genéticas entre los efectos directos $\left(\mathbf{r}_{a a}\right)$, los maternos $\left(\mathbf{r}_{m m}\right)$, y los directos con maternos $\left(r_{a m}\right)$. Las $h_{a}^{2}$ oscilaron de 0.18 a 0.30 , y las $h_{m}^{2}$ de 0.07 a 0.39 ; lo cual indica una probable respuesta a la selección en PEA y TAC. Todas las correlaciones genéticas fueron positivas en un intervalo de 0.09 a 0.87 , con excepción de $r_{a a}$ entre PEA y TAC (-0.57); este antagonismo genético indica que los individuos con tasas altas de crecimiento no son los de mayor peso adulto.

Palabras clave: Correlación genética, raza sintética, ganadería tropical, modelos no lineales, heredabilidad.

ABSTRACT. The aim of this study was to estimate genetic parameters for mature weight (MW) and growth rate (GR) derived from the nonlinear Brody model, and analyze the possible genetic relationships between these variables and weaning weight $(\mathrm{WW})$. The information analyzed was $\mathrm{MW}(\mathrm{kg} ; \mathrm{n}=994), \mathrm{GR}\left(\mathrm{kg} \mathrm{kg}^{-1}\right.$ month $\left.^{-1}, \mathrm{n}=994\right)$, and $W W$ adjusted to $240 \mathrm{~d}(\mathrm{~kg} ; \mathrm{n}=1506)$; the mean values and standard deviation $( \pm)$ for $\mathrm{MW}, \mathrm{GR}$ and WW were 419.1 $\pm 94.4,0.0081 \pm 0.0039$ and $206.8 \pm 34.1$, respectively; the pedigree was composed of 2504 records. A multivariate analysis was performed (MW, GR and WW) from a mixed model that included as fixed effects the contemporary groups and the covariate age of the cow at calving (linear and quadratic), and as random ones the direct and maternal, maternal permanent environment, and residual genetic effects. Direct $\left(h_{a}^{2}\right)$ and maternal $\left(h_{m}^{2}\right)$ heritability, as well as the genetic correlations between direct $\left(\boldsymbol{r}_{a a}\right)$, maternal $\left(\boldsymbol{r}_{m m}\right)$, and direct with maternal effects $\left(\boldsymbol{r}_{a m}\right)$ were estimated. The $h_{a}^{2}$ varied from 0.18 to 0.30 , and the $h_{m}^{2}$ from 0.07 to 0.39 , indicating that there could be a response to the selection in MW and GR. All genetic correlations were positive in an interval of 0.09 to 0.87 , except the $r_{a a}$ between MG and GR (-0.57), this genetic antagonism indicates that individuals with high growth rates do not have the highest mature weight.

Key words: Genetic correlation, synthetic breed, tropical livestock, nonlinear models, heritability 


\section{INTRODUCCIÓN}

La curva de crecimiento de bovinos para carne usualmente se determina mediante las mediciones y ajustes del peso vivo a edades diferentes, considerados como datos longitudinales o medidas repetidas de la misma característica (Meyer 1999, Forni et al. 2007). El análisis del peso vivo ajustado a determinadas edades, supone que todos los animales presentan una curva similar de crecimiento, al mismo tiempo que los efectos genéticos y ambientales, así como las varianzas y covarianzas de los efectos aleatorios son constantes en el intervalo ajustado (Meyer 1998). Otra alternativa es la implementación de modelos no lineales (MNL) que describen el proceso de crecimiento en el tiempo en función de un número reducido de parámetros de crecimiento con una interpretación biológica (Domínguez-Viveros et al. 2013). El conocimiento de los parámetros de crecimiento derivados de los MNL permite implementar programas específicos de alimentación, manejo y comercialización (López de Torre et al. 1992, Mara y Moura 1999); así como definir esquemas de mejoramiento genético con el fin de modificar la curva de crecimiento (Kaps et al. 1999, Ibáñez-Escriche y Blasco 2011). En la curva de crecimiento de bovinos para carne, el peso al destete (PD) es una medida de suma importancia; en este punto, el individuo alcanza entre el 25 y $35 \%$ de su peso final, que es un indicador de la eficiencia del sistema de producción, así como una medida del potencial de crecimiento del becerro y de las cualidades de la madre (Arango y Van Vleck 2002, Jakubec et al. 2003). El PD es el principal criterio de selección de los criadores de bovinos para carne, por lo que los posibles cambios derivados de una respuesta a la selección, pueden repercutir en la forma de la curva de crecimiento (BIF 2010).

La raza Tropicarne, es una raza sintética de bovinos para carne que se desarrolló en la región tropical del Golfo de México; con una composición racial de $63 \%$ Barzona, 23\% Senepol, $9 \%$ Brahman y $5 \%$ Charolais (Ruiz-Flores et al. 2006). La proporción de heterosis retenida es de aproximadamente $54 \%$; más la complementariedad de las razas utilizadas en su formación, le confieren características de desempeño y adaptación bajo las condiciones ambientales del trópico del Golfo de México, donde la alimentación se basa en el pastoreo de gramíneas forrajeras (Domínguez-Viveros et al. 2003a, NúñezDomínguez et al. 2010). En donde las condiciones extremas de temperatura y humedad, y la incidencia de parásitos (internos y externos), ejercen efectos directos e indirectos en el crecimiento y la producción (Burrow 2012, Galukande et al. 2013).

En bovinos Tropicarne se ha evaluado genéticamente el crecimiento con información de peso al nacer, al destete, al año y a los 18 meses de edad, mediante el modelo animal con efectos maternos y en ciertos casos multivariados (DomínguezViveros et al. 2003b, Ramírez-Valverde et al. 2007); además, se ha caracterizado el crecimiento con MNL (Domínguez-Viveros et al. 2013), se han generado parámetros de crecimiento con el modelo de Brody, como el peso adulto o asintótico y la pendiente de la curva relativa a la tasa de crecimiento o de madurez. Con el fin de evaluar la posible incorporación de los parámetros de crecimiento a los programas de mejoramiento genético de bovinos Tropicarne, bajo la suposición de que existe variabilidad genética relacionada con el PD, el objetivo del presente estudio fue estimar parámetros genéticos para el peso adulto y la tasa de crecimiento, además de analizar las relaciones genéticas de estas variables con el PD.

\section{MATERIALES Y MÉTODOS}

La base de datos, correspondió a 994 registros de peso adulto o asintótico en kg (PEA), y 994 de tasa de crecimiento o de madurez $\mathrm{kg}^{-1}$ mes $^{-1}$ (TAC) de bovinos Tropicarne de ambos sexos. Los datos fueron derivados del modelo no lineal de Brody; a partir de los resultados publicados por Domínguez-Viveros et al. (2013), este modelo tuvo un mejor ajuste para caracterizar el crecimiento de bovinos Tropicarne. El peso al destete en $\mathrm{kg}$ (PD) se ajustó a 240 d. La información genealógica, correspondiente al pedigrí, se integró por 2504 individuos, los cuales formaron la matriz de las relaciones genéticas aditivas $(A)$. 
Para la estimación de los componentes de varianza, se realizó un análisis multivariado, incluyendo de manera conjunta el PEA, la TAC y el $\mathrm{PD}$, a partir del modelo mixto $\mathrm{y}=\mathrm{X} \beta+\mathrm{Za}+$ $\mathrm{Mm}+\mathrm{Wp}+e$; donde: $\mathrm{y}$, es el vector de registros del PEA, la TAC y el PD; $\beta$, es el vector de efectos fijos que incluyó a los grupos contemporáneos definidos por las combinaciones año - época de nacimiento y sexo; además de la edad de la vaca al parto como covariable en las funciones lineal y cuadrática; $a$, es el vector de los efectos aleatorios genéticos aditivos directos; $m$, es el vector de los efectos aleatorios genéticos aditivos maternos; $p$, es el vector de los efectos aleatorios maternos de ambiente permanente, no correlacionados; $e$, es el vector de los efectos residuales; X, Z, M y W son las matrices de incidencia, que asocian a los efectos en los vectores correspondientes con y. Las suposiciones del modelo fueron: $\mathrm{E}[\mathrm{y}]=\mathrm{X} \beta$ y $\mathrm{E}[a]=\mathrm{E}[m]$ $=\mathrm{E}[p]=\mathrm{E}[e]=0$; los componentes de varianza y covarianza estimados fueron: $\mathrm{A} \sigma^{2} a_{i}=$ varianzas de efectos genéticos aditivos directos $(i=1,2$ y 3); $A \sigma m_{i}=$ varianzas de efectos genéticos aditivos maternos ( $\mathrm{j}=1,2$ y 3 ); $\mathrm{A} \sigma a_{i} a_{j}=$ covarianzas entre efectos genéticos aditivos directos; $\mathrm{A} \sigma a_{i} m_{i}$ $=$ covarianzas entre efectos genéticos aditivos directos y maternos; $\mathrm{A} \sigma m_{i} m_{j}=$ covarianzas entre efectos genéticos aditivos maternos; $\sigma^{2} p_{i}=$ varianzas de efectos maternos de ambiente permanente; $\sigma^{2} e_{i}=$ varianzas residuales; $\sigma e_{i} e_{j}=$ covarianzas entre efectos residuales. Los análisis estadísticos se realizaron con la metodología del mejor predictor de los efectos aleatorios y mejor estimador de los efectos fijos, a través del modelo animal (Mrode 2014, Martínez et al. 2012). Para e análisis multivarido se utilizó el software MTDFREML el cual emplea el procedimiento de máxima verosimilitud libre de derivadas (Boldman et al. 1995). A partir de los componentes de varianza y covarianza de los datos analizados, se estimaron las correlaciones fenotípicas $\left(r_{f f}\right)$; posteriormente, para los efectos aleatorios se generaron los parámetros genéticos a proporcionalidad: heredabilidad de efectos genéticos directos $\left(\mathrm{h}_{a}^{2}\right)$; heredabilidad de efectos genéticos maternos $\left(h_{m}^{2}\right)$; correlaciones genéticas entre efectos directos $\left(r_{a a}\right)$; correlaciones genéticas entre efectos maternos $\left(r_{m m}\right)$; y correlaciones genéticas entre efectos directos y maternos $\left(r_{a m}\right)$.

\section{RESULTADOS}

El valor promedio y la desviación estándar ( \pm ) para PEA, TAC y PD $(\mathrm{kg})$ fue de $419.1 \pm$ $94.4,0.0081 \pm 0.0039$ y $206.8 \pm 34.1$, respectivamente. Las estimaciones de heredabilidades fueron de media a alta magnitud (Tabla 1 ), la $h_{a}^{2}$ fluctúo de 0.18 a 0.30 , y la $\mathrm{h}_{m}^{2}$ de 0.07 a 0.39 ; estos resultados señalan que puede haber una respuesta a la selección en estos parámetros de crecimiento, con posibles consecuencias en el formato de la curva de crecimiento. Las $r_{f f}$ de PD con PEA y TAC fueron de 0.28 y 0.58 , respectivamente; además, la $r_{f f}$ entre PEA y TAC fue de -0.41. Las correlaciones genéticas en su mayoría fueron positivas, con excepción de $r_{a a}$ entre PEA y TAC que fue negativa y de mediana magnitud (-0.57); las $r_{f f}$ y $r_{a a}$ coincidieron en magnitud y signo a través de las variables analizadas. Las $r_{a m}$ fluctuaron de 0.09 a 0.87 , con un valor promedio de 0.52 ; asimismo, las $\mathbf{r}_{m m}$ fueron igual o mayor a 0.70 .

\section{DISCUSIÓN}

Los intervalos en las estimaciones de heredabilidad, publicados por estudios similares, coinciden con los resultados del presente estudio. DeNise y Brinks (1985) y Jenkins et al. (1991), con procedimientos a partir del análisis de varianza publicaron estimaciones de $h_{a}^{2}$ en un intervalo de 0.21 a 0.61 para PEA y de 0.20 a 0.75 para TAC, derivados del modelo de Brody en diversas razas de bovinos para carne Bos taurus. Al respecto Bullock et al. (1993), Northcutt y Wilson (1993) y Kaps et al. (2000), con base en modelos mixtos y procedimientos de máxima verosimilitud, obtuvieron estimaciones de $\mathrm{h}_{a}^{2}$ en un intervalo de 0.45 a 0.52 para PEA y de 0.15 a 0.24 para TAC en bovinos Angus y Hereford. Mientras que Mara y Moura (1999), Garnero et al. (2005) y del Valle et al. (2006), con análisis similares, reportaron es- 
Tabla 1. Estimaciones de heredabilidades y correlaciones genéticas ( \pm error estándar) para peso adulto (PEA), tasa de crecimiento (TAC) y peso al destete (PD) en bovinos Tropicarne .

\begin{tabular}{|c|c|c|c|c|c|c|}
\hline & \multicolumn{3}{|c|}{ Efectos genéticos directos } & \multicolumn{3}{|c|}{ Efectos genéticos maternos } \\
\hline & $\mathrm{PEA}_{a}$ & $\mathrm{TAC}_{a}$ & $\mathrm{PD}_{a}$ & $\mathrm{PEA}_{m}$ & $\mathrm{TAC}_{m}$ & $\mathrm{PD}_{m}$ \\
\hline $\mathrm{PEA}_{a}$ & $0.23 \pm 0.11$ & & & & & \\
\hline $\mathrm{TAC}_{a}$ & $-0.54 \pm 0.20$ & $0.30 \pm 0.15$ & & & & \\
\hline $\mathrm{PD}_{a}$ & $0.34 \pm 0.10$ & $0.57 \pm 0.14$ & $0.18 \pm 0.09$ & & & \\
\hline $\mathrm{PEA}_{m}$ & $0.47 \pm 0.12$ & $0.22 \pm 0.08$ & $0.62 \pm 0.18$ & $0.07 \pm 0.05$ & & \\
\hline $\mathrm{TAC}_{m}$ & $0.79 \pm 0.18$ & $0.09 \pm 0.11$ & $0.81 \pm 0.15$ & $0.70 \pm 0.06$ & $0.18 \pm 0.07$ & \\
\hline $\mathrm{PD}_{m}$ & $0.49 \pm 0.12$ & $0.41 \pm 0.07$ & $0.79 \pm 0.14$ & $0.79 \pm 0.13$ & $0.87 \pm 0.15$ & $0.39 \pm 0.12$ \\
\hline
\end{tabular}

timaciones de $\mathrm{h}_{a}^{2}$ de 0.09 a 0.42 para PEA y de 0.13 a 0.42 para TAC en bovinos Nelore y Chianina. Para $h_{m}^{2}$ en PEA, Rumph et al. (2002) formularon estimaciones en un intervalo de 0.09 a 0.18 en bovinos Hereford. Por otro lado, las estimaciones de $h_{a}^{2}$ y $h_{m}^{2}$ para PD son superiores a los valores promedio reportados por Zerlotti et al. (1995) y Ríos (2008) para PD y otras variables de crecimiento en diversas razas de bovinos para carne. Dado que Tropicarne es una raza sintética, las diferencias en variabilidad genética con respecto a otras poblaciones, se pueden atribuir a los efectos genéticos aportados por las razas involucradas en su formación (Bennett y Gregory 1996, Vergara et al. 2009). Para bovinos Tropicarne, las estimaciones de $h_{a}^{2}$ y $\mathrm{h}_{m}^{2}$ para PD en el presente estudio fueron superiores a las reportadas por Domínguez-Viveros et al. (2003b) y Ramírez-Valverde et al. (2007). Estas diferencias se pueden atribuir a las ventajas del análisis multivariado (Mrode 2014), así como una mejor estructura de los datos y de las relaciones de parentesco en la matriz de parentesco (Clément et al. 2001), que permitan estimar las varianzas y covarianzas genéticas de mejor manera.

Con relación a los resultados en las correlaciones fenotípicas y genéticas, las $r_{f f}$ vs $r_{a a}$ fueron similares en magnitud y signo, lo cual puede indicar que las diferencias fenotípicas pueden ser producto de una respuesta a la selección; y las estimaciones en $r_{m m}$ y $r_{a m}$ se pueden atribuir a que no hay oposición entre los efectos genéticos directos y maternos (Bennett y Gregory 1996). Estimaciones de $r_{a a}$ negativas y de media a alta magnitud en- tre PEA y TAC fueron reportadas por Jenkins et al. (1991), Kaps et al. (1999) y Garnero et al. (2005). Este antagonismo genético puede indicar que los individuos con altas TAC no necesariamente son los de mayor PEA, o los individuos con mayores PEA no necesariamente son producto de altas TAC; incrementos en el PEA del animal, especialmente en las hembras, pueden afectar la rentabilidad del sistema de producción vaca - becerro (Arango y Van Vleck 2002, Rumph et al. 2002). El aumento del PEA resulta en un incremento en los requerimientos para mantenimiento, reproducción y producción, y no necesariamente son los individuos más productivos. Al respecto, Núñez-Domínguez et al. (2010) reportaron que las vacas Tropicarne con potencial genético para peso corporal grande produjeron becerros más pesados al destete que las pequeñas, pero tuvieron tasas de parición y de destete similares a las pequeñas y medianas; sin embargo, las vacas pequeñas fueron más productivas que las grandes al momento del destete, cuando se expresó el peso del becerro como proporción del peso de la vaca.

La $r_{a a}$ entre PD y TAC (0.57), indica que los becerros con altos PD provienen de altas TAC, lo cual se puede atribuir al período pre destete, donde el becerro expresa la mayor tasa de crecimiento, alcanzando gran parte del peso adulto (DomínguezViveros et al. 2003a). Aunado a esto, en el presente estudio el PD conformó $49.3 \%$ del PEA; además, el crecimiento predestete se puede definir con una función lineal de primer orden (Villalba et al. 2000), y el ajuste del PD se realiza con base en la ganancia diaria predestete (BIF 2010). Por otro lado, 


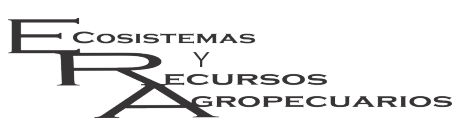

el crecimiento posdestete es el principal indicador del potencial de crecimiento del animal. Se puede evaluar por la ganancia diaria o total, o por el peso vivo a determinado punto o edad, como un resumen de los efectos genéticos y ambientales (Meyer 1999, Cucco et al. 2010); no obstante, los efectos genéticos que infieren en el posdestete, como es el caso del PEA, también están asociados con el PD, lo que trasciende en una $r_{a a}$ positiva (Groeneveld et al. 1998, Meyer 1999). Con el objetivo de obtener curvas de mejor pendiente sin incrementos en el peso adulto, Arango y Van Vleck (2002) indican que la selección sobre velocidad de crecimiento es una alternativa, para alcanzar lo antes posible el peso comercial. Para utilizar el PD como criterio de selección, se puede abordar como proporción o parte porcentual del PEA, con cambios en la edad al destete para aprovechar la tasa de crecimiento predestete. Consecuentemente, los incrementos de PD como proporción del PEA, es una opción para alcanzar lo antes posible el peso adulto. Con el objetivo de alterar la forma de la curva de crecimiento animal, en la evaluación de diversas estrategias de selección considerando la variabilidad genética de los parámetros de crecimiento derivados de los MNL; así como las relaciones genéticas con otras variables de importancia fisiológica y económica (Piles et al. 2003, Forni et al. 2007, Ibáñez-Escriche y Blasco 2011).

Las estimaciones positivas de $r_{m m}$ podrían indicar que el potencial de las madres para producir leche trasciende de manera positiva en el desempeño y el crecimiento de los becerros (Lee 2001). La magnitud e importancia de los efectos maternos en el crecimiento de los bovinos para carne difiere a través del tiempo, características y genotipos; no incluirlos en los modelos estadísticos puede afectar las estimaciones de la varianza genética aditiva directa, y por lo tanto, la respuesta a la selección (Bijma 2006). De los efectos maternos, la variación en los requerimientos de mantenimiento puede explicar hasta $23 \%$ de las diferencias en la producción de leche (Arango y Van Vleck 2002). Todas las estimaciones de $r_{a m}$ fueron positivas (Cuadro 1 ), con un valor promedio de 0.52 en un intervalo de 0.09
Dominguez-Viveros et al. Parámetros genéticos en bovinos Tropicarne Esosist. Recur. Agropec. 4(10):81-88,2017

a 0.81; estos resultados pueden indicar que no hay un antagonismo entre los genes que establecen la habilidad de la cría para el crecimiento y los genes que determinan el potencial para la producción de leche de las madres (Lee 2001, David et al. 2016). Estos resultados difieren a lo reportado por Lee y Pollak (2002) y Ríos (2008), quienes publicaron estimaciones de $r_{a m}$ negativas para PD, de mediana a alta magnitud en razas de bovinos para carne. La $r_{a m}$ positiva para PD se puede asociar a la forma de la curva de crecimiento; que se ajustó al modelo de Brody (Domínguez-Viveros et al. 2013) que no presenta un punto de inflexión. La diferencia en los MNL radica en la posición del punto de inflexión de la curva, que denota un cambio en la velocidad y el modo de crecimiento; la edad al punto de inflexión está dentro del periodo pre destete, por consiguiente, ese cambio en la velocidad y modo de crecimiento se puede manifestar en el signo negativo de la $r_{a m}$ en PD. El modelo de Brody carece de punto de inflexión, considera a la velocidad de crecimiento proporcional al crecimiento que queda por efectuar, las tasas de crecimiento disminuyen a medida que aumenta el peso y la edad, y este comportamiento lineal se puede expresar en una $r_{a m}$ positiva.

\section{CONCLUSIONES}

Las estimaciones de las heredabilidades directas y maternas fueron de mediana a alta magnitud, como indicación de que existe variabilidad genética. Esto sugiere que los parámetros de crecimiento peso adulto y tasa de crecimiento, derivados del modelo de Brody en bovinos Tropicarne, pueden incluirse en un programa de mejoramiento genético y esperar una respuesta a la selección. Con base en la estimación negativa de la correlación genética entre el peso adulto y la tasa de crecimiento, los animales con mayores tasas de crecimiento, no necesariamente son los más pesados en edad adulta, lo que pudiera ser conveniente al seleccionar para mayores tasas de crecimiento, manteniendo un peso adulto constante. 


\section{AGRADECIMIENTOS}

A los criadores de bovinos Tropicarne el pro- porcionar la información productiva y genealógica para realizar el presente estudio.

\section{LITERATURA CITADA}

Arango JA, Van Vleck LD (2002) Size of beef cows: early ideas, new developments. Genetics and Molecular Research 1: 51-63.

Bennett GL, Gregory KE (1996) Genetic (co)variances among birth weight, 200-day weight, and postweaning gain in composites and parental breeds of beef cattle. Journal of Animal Science 74: 2598-2611.

BIF (2010) Guidelines for Uniform Beef Improvement Program. 9th Edition. Beef Improvement Federation. Colby, KS. USA. 185p.

Bijma P (2006) Estimating maternal genetic effects in livestock. Journal of Animal Science 84: 800-806.

Boldman KG, Kriese LA, Van Vleck LD, Van Tassell CP, Kachman SD (1995) A manual for use of MTDFREML. A set of programs to obtain estimates of variances and covariances. USDA - ARS, Clay Center, NE. USA. 114p.

Bullock KD, Bertrand JK, Benyshek LL (1993) Genetic and environmental parameters for mature weight and other growth measures in polled Hereford cattle. Journal Animal Science 71: 1737-1741.

Burrow HM (2012) Importance of adaptation and genotype $x$ environment interactions in tropical beef breeding systems. Animal 6: 729-740.

Clément V, Bibé B, Verrier É, Elsen JM, Manfredi E, Bouix J, et al. (2001) Simulation analysis to test the influence of model adequacy and data structure on the estimation of genetic parameters for traits with direct and maternal effects. Genetic Selection Evolution 33: 369-395.

Cucco DC, Ferraz JBS, Eler JP, Baliero JCC, Mattos EC, Varona L (2010) Genetic parameters for postweaning traits in Braunvieh cattle. Genetics and Molecular Research 9: 545-553.

David I, Bouvier F, Banville M, Canario L, Flatres-Grall L, Balmisse E, et al. (2016) The direct-maternal genetic correlation has little impact on genetic evaluations. Journal of Animal Science 93: 5639-5647.

del Valle GA, Righetti MC, José GR, Nunes de OH, Barbosa LR (2006) Genetic trends in the expected progeny difference of the asymptotic weight of Nelore females. Genetics and Molecular Biology 29: 648-652.

DeNise KRS, Brinks JS (1985) Genetic and environmental aspects of the growth curve parameters in beef cows. Journal of Animal Science 61: 1431-1440.

Domínguez-Viveros J, Núñez-Domínguez R, Ramírez-Valverde R, Ruiz-Flores A (2003a) Influencias ambientales e índice de constancia para características de crecimiento en ganado bovino Tropicarne. Técnica Pecuaria en México 41: 1-18.

Domínguez-Viveros J, Núñez-Domínguez R, Ramírez-Valverde R, Ruiz-Flores A (2003b) Evaluación genética de variables de crecimiento en bovinos Tropicarne: I. Selección de modelos. Agrociencia 37: 323-335.

Domínguez-Viveros J, Rodríguez-Almeida FA, Núñez-Domínguez R, Ramírez-Valverde R, Ortega-Gutiérrez JA, Ruiz-Flores A (2013) Ajuste de modelos no lineales y estimación de parámetros de crecimiento en bovinos Tropicarne. Agrociencia 47: 25-34. 
Forni S, Piles M, Blasco A, Varona L, Oliveira HN, Lôbo RB, et al. (2007) Analysis of beef cattle longitudinal data applying a nonlinear model. Journal of Animal Science 85: 3189-3197.

Galukande E, Mulindwa H, Wurzinger M, Roschinsky R, Mwai AO, Sölkner J (2013) Cross-breeding cattle for milk production in the tropics: achievements, challenges and opportunities. Animal Genetics Research 52: $111-125$.

Garnero AV, Marcondes CR, Bezerra LAF, Oliveira HN, Lôbo RB (2005) Parâmetros genéticos de taxa de maturação e do peso assintótico de fêmeas de raçã Nelore. Arquivo Brasileiro de Medicina Veterinária e Zootecnia 57: 652-662.

Groeneveld E, Mostert BE, Rust T (1998) The covariance structure of growth traits in the Afrikanerbeef population. Livestock Production Science 55: 99-107.

Ibáñez-Escriche N, Blasco A (2011) Modifying growth curve parameters by multitrait genomic selection. Journal of Animal Science 89: 661-668.

Jakubec V, Schlote W, Ríha J, Majzlík I (2003) Comparison of growth traits of eight beef cattle breeds in the Czech Republic. Archive Tierz Dummerstorf 46: 143-153.

Jenkins TG, Kaps M, Cundiff LV, Ferrell CL (1991) Evaluation of between - and within - breed variation in measures of weight - age relationships. Journal of Animal Science 69: 3118-3128.

Kaps M, Herring WO, Lamberson WR (1999) Genetic and environmental parameters for mature weight in Angus cattle. Journal of Animal Science 77: 569-574.

Kaps M, Herring WO, Lamberson WR (2000) Genetic and environmental parameters for trait derived from the Brody growth curve and their relationships with weaning weight in Angus cattle. Journal of Animal Science 78: 1436-1442.

Lee C (2001) On the negative estimates of direct and maternal genetic correlation - A review. AsianAustralasian Journal of Animal Sciences 15: 1222-1226.

Lee C, Pollak EJ (2002) Genetic antagonism between body weight and milk production in beef cattle. Journal of Animal Science 80: 316-321.

López de Torre G, Candotti JJ, Reverter A, Bellido MM, Vasco P, García LJ, et al. (1992). Effects of growth curve parameters on cow efficiency. Journal of Animal Science 70: 2688-2672.

Mara CS, Moura DFA (1999) Description and comparison of growth parameters in Chianina and Nelore cattle breeds. Genetics and Molecular Biology 22: 187-196.

Martínez CA, Manrique C, Elzo M (2012) La evaluación genética de vacunos: una percepción histórica. Revista Colombiana de Ciencias Pecuarias 25: 293-311.

Meyer K (1998) Estimating covariance functions for longitudinal data using a random regression model. Genetic Selection Evolution 30: 221-240.

Meyer K (1999) Estimate of genetic and phenotypic covariance functions for post weaning growth and mature weight of beef cows. Journal of Animal Breeding and Genetic 116: 181-205.

Mrode RA (2014) Linear Models for the Prediction of Animal Breeding Values. 2th edition. CAB International, Wallingford Oxon, UK. 344p.

Northcutt SL, Wilson DE (1993) Genetic parameter estimate and expected progeny differences for mature size in Angus cattle. Journal of Animal Science 71: 1148-1153. 
Núñez-Domínguez R, Hernández-Rodríguez BA, Ramírez-Valverde R, Ruíz-Flores A, García-Muñiz JG, LópezOrdaz R (2010) Productividad de vacas Tropicarne con diferente potencial genético para peso corporal al primer servicio. Revista Científica FCV-LUZ 20: 640-648.

Piles M, Gianola D, Varona L, Blasco A (2003) Bayesian inference about parameters of a longitudinal trajectory when selection operates on a correlated trait. Journal of Animal Science 81: 2714-2724.

Ramírez-Valverde R, Hernández-Álvarez OC, Núñez-Domínguez R, Ruiz-Flores A, García-Muñiz JG (2007) Análisis univariado vs multivariado en la evaluación genética de variables de crecimiento en dos razas bovinas. Agrociencia 41: 271-282.

Ríos UA (2008) Estimadores de parámetros genéticos para características de crecimiento predestete de bovinos. Revisión. Técnica Pecuaria en México 46: 37-67.

Ruiz-Flores A, Núñez-Domínguez R, Ramírez-Valverde R, Domínguez-Viveros J, Mendoza-Domínguez M, Martínez-Cuevas E (2006) Niveles y efectos de la consanguinidad en variables de crecimiento y reproductivas en bovinos Tropicarne y Suizo Europeo. Agrociencia 40: 289-301.

Rumph JM, Koch RM, Gregory KE, Cundiff LV, Van Vleck LD (2002) Comparison of models for estimation of genetic parameters for mature weight of Hereford cattle. Journal of Animal Science 80: 583-590.

Vergara OD, Elzo MA, Ceron-Muñoz MF, Arboleda EM (2009) Weaning weight and post-weaning gain genetic parameters and genetic trends in a Blanco Orejinegro-Romosinuano-Angus-Zebu multibred cattle population in Colombia. Livestock Science 124: 156-162.

Villalba D, Casasus I, Sanz A, Estany J, Revilla R (2000) Preweaning growth curves in Brown Swiss and Pirenaica calves with emphasis on individual variability. Journal of Animal Science 78: 1132-1140.

Zerlotti MME, Barbosa LR, de los Reyes BA (1995) Parámetros genéticos de crecimiento en cebuinos de carne. Archivos Latinoamericanos de Producción Animal 3: 45-89. 